\title{
Exploring the Effects of Observed Physicality Conflicts on Real-Virtual Human Interaction in Augmented Reality
}

\author{
Kangsoo Kim \\ University of Central Florida \\ Orlando, Florida, United States \\ kskim@knights.ucf.edu
}

\author{
Gerd Bruder \\ University of Central Florida \\ Orlando, Florida, United States \\ bruder@ucf.edu
}

\author{
Greg Welch \\ University of Central Florida \\ Orlando, Florida, United States \\ welch@ucf.edu
}

\begin{abstract}
Augmented reality (AR) enables the illusion of computer-generated virtual objects and humans co-existing with us in the real world. Virtual humans (VHs) in AR can further induce an illusion of physicality in the real world due to their form of presentation and their behavior, such as showing awareness of their surroundings. However, certain behaviors can cause a conflict that breaks this illusion, for example, when we see a VH passing through a physical object.

In this paper we describe a human-subject study that we performed to test the hypothesis that participants experience higher copresence in conflict-free circumstances, and we investigate the magnitude of this effect and behavioral manifestations. Participants perceived a social situation in a room that they shared with a $\mathrm{VH}$ as seen through a HoloLens head-mounted display. The behavior of the VH either caused conflicts with (occupied the same space as) physical entities, or avoided them. Our results show that the conflicts in physicality significantly reduced subjective reports of copresence. Moreover, we observed that participants were more likely to cause a conflict (occupy the same space as) virtual entities in case the VH had avoided the conflict. We discuss implications for future research and shared AR setups with real-virtual human interactions.
\end{abstract}

\section{CCS CONCEPTS}

- Human-centered computing $\rightarrow$ User studies; Mixed / augmented reality; • Computing methodologies $\rightarrow$ Mixed / augmented reality; $\bullet$ Applied computing $\rightarrow$ Psychology;

\section{KEYWORDS}

Augmented reality, virtual humans, copresence, physicality

\section{ACM Reference Format:}

Kangsoo Kim, Gerd Bruder, and Greg Welch. 2017. Exploring the Effects of Observed Physicality Conflicts on Real-Virtual Human Interaction in Augmented Reality. In Proceedings of VRST '17. ACM, New York, NY, USA, 7 pages. https://doi.org/10.1145/3139131.3139151

Permission to make digital or hard copies of all or part of this work for personal or classroom use is granted without fee provided that copies are not made or distributed for profit or commercial advantage and that copies bear this notice and the full citation on the first page. Copyrights for components of this work owned by others than ACM must be honored. Abstracting with credit is permitted. To copy otherwise, or republish, to post on servers or to redistribute to lists, requires prior specific permission and/or a fee. Request permissions from permissions@acm.org.

VRST '17, November 8-10, 2017, Gothenburg, Sweden

(C) 2017 Association for Computing Machinery.

ACM ISBN 978-1-4503-5548-3/17/11. \$15.00

https://doi.org/10.1145/3139131.3139151

\section{INTRODUCTION}

Compared to virtual reality (VR), where sensations of the real world are replaced by those of a computer-generated virtual environment, in augmented reality (AR) virtual sensations are superimposed upon or composited with the real world [Azuma 1997]. Typically, a user wears a head-mounted display (HMD) that provides a tracked (position and orientation) stereoscopic view of the real world, with superimposed computer-generated graphics that are related and registered to the real world. Much previous research in AR was focused on the seamless visual integration of virtual objects into the real world, such as by providing believable indirect lighting [Debevec 2005] or improving the registration of real and virtual objects in the augmented view of the real world [Azuma and Bishop 1994]. Moreover, for dynamic virtual objects or virtual humans (VHs) it is generally considered important to give an illusion of physicality by simulating virtual gravity and realistic physical constraints [Breen et al. 1996; Chae and Ko 2008]. Such virtual humans could either be computer-controlled agents or human-controlled avatars.

While most AR environments try to provide such a consistent and conflict-free augmented view, it does happen that VHs pass through physical objects, and sometimes it is unavoidable. For instance, if a virtual agent has a pre-programmed task to enter a room, but the physical door to the room is closed, then it is at the dilemma to either not be able to fulfill its programming or induce a conflict by passing through the door (see Figure 1). Such behavior could elevate the virtual human over real humans due to its "super powers," or lower them by highlighting their non-physicality. In any case, it is likely that such behavior would alter social interactions between real and virtual humans.

In this paper we describe a human-subject study in which we had participants experience different social interactions with a $\mathrm{VH}$ in a physical room. The VH was presented on a HoloLens HMD and either followed the rules of physicality or disregarded them (like a "ghost") by moving through physical objects (i.e., occupying the same space). We analyzed subjective responses for the participants' sense of copresence and behavioral data. We discuss the results with respect to human behavior during social interaction in AR.

This paper is structured as follows: Section 2 gives an overview of related work. Section 3 describes the experiment which we conducted to investigate the sense of copresence with respect to the VH's behavior. Section 4 presents the results of our experiment, which are discussed in Section 5. Section 6 concludes the paper.

\section{RELATED WORK}

This section provides an overview of related work on VHs in AR and the concept of social presence and copresence. 


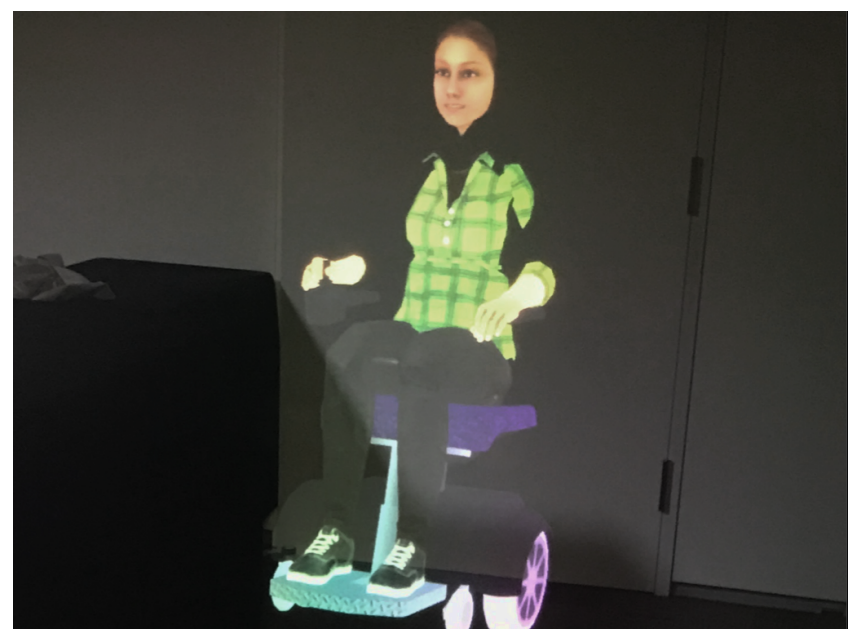

Figure 1: Photo taken through a Microsoft HoloLens optical see-through head-mounted display showing a virtual human (in a wheelchair) entering the room by driving through a closed physical door in a "ghost-like" fashion, i.e., the virtual human's more distant body parts are occluded by the door.

\subsection{Virtual Humans in AR}

Holz et al. [Holz et al. 2011, 2009] provide a survey of various forms of agents in a fully physical, a fully virtual, or a mixed reality (MR) environment in the context of social interaction. They further detail the benefits and issues with social interaction with virtual agents. Obaid et al. [Obaid et al. 2011] conducted a study comparing the participants' voice level while interacting with agents in AR and VR, and found that in both conditions their voice level compensated for the distance to the agent. Interestingly, the effect was stronger in $\mathrm{AR}$, which might be explained by participants perceiving the distance between themselves and the AR agent with less perceptual error [Loomis and Knapp 2003]. In a different study, Obaid et al. [Obaid et al. 2012] evaluated the relationship between the physiological arousal of real humans and an agent's behavior in AR associated with cultural differences, such as in personal space and gaze, and suggested that mutual gaze had a higher impact on one's sense of arousal than the interpersonal distance.

Focusing on applications of VHs in AR, Magnenat-Thalmann et al. [Magnenat-Thalmann et al. 2008] surveyed various fields that would benefit from employing VHs, such as industrial training and cultural heritage guidance. Torre et al. [Torre et al. 2000] superimposed an AR human that could play a checker game with real human users in a shared MR environment. Jo et al. [Jo et al. 2015] developed an AR tele-presence framework using an avatar in AR controlled by a remote user, and discussed how to maintain the avatar's realism in the physical place by adapting its motion considering the surrounding physical objects. Pejsa et al. [Pejsa et al. 2016] developed a life-sized telepresence system called "Room2Room." This approach employs digital projectors to display a remote participant in the local room. It leverages the available physical affordances of the rooms, and maps local/remote participants to physical locations using either a predefined approach where the mapping is specified a priori, or an approach where the mapping is determined on an adhoc basis, depending on the participant's location, movement, etc. Furthermore, Microsoft introduced a game called "Fragments" [Microsoft 2017a] where people can see and interact with VHs in AR through a HoloLens HMD. While it is unclear whether the VHs in every instance of the game will maintain compliance with the physical surroundings, Microsoft Developer guidelines for HoloLens "Spatial Mapping" [Microsoft 2017b] mention the need for visual conflict-free real-virtual relationships and interactions, for objects and humans.

\subsection{Social and Co-Presence with Virtual Humans}

Two of the most common measures for the effectiveness of VHs in conveying the illusion of being real are social presence and copresence. The former denotes the sense of "being socially connected" and the latter "being together." While there is no universal agreement on the definitions for social and co-presence, Harms and Biocca [Harms and Biocca 2004] consider co-presence as one of several sub-dimensions that embody social presence, and Blascovich et al. [Blascovich 2002; Blascovich et al. 2002] define social presence both as a "psychological state in which the individual perceives himself or herself as existing within an interpersonal environment" and "the degree to which one believes that he or she is in the presence of, and dynamically interacting with, other veritable human beings." Both definitions are related to presence, which denotes the sense of "being there." In particular, Slater [Slater 2009] introduced the concepts of place illusion and plausibility illusion which together define presence. According to Slater [Slater 2009], the latter refers to the illusion that "the scenario being depicted is actually occurring" and that it requires a "credible scenario and plausible interactions between the participant and objects and virtual characters in the environment."

Different characteristics of the real and virtual humans have been observed to influence the real human's sense of social and co-presence during interaction. For example, Fox et al. [Fox et al. 2014] evaluated relationships between the perceived agency of VHs and measures such as questionnaires, physiological responses and proxemics. When participants perceived that a $\mathrm{VH}$ was controlled by a real human (an avatar), it was more influential than if it was perceived to be controlled by a computer algorithm (an agent). Nowak and Biocca [Nowak and Biocca 2003] did not find any agency effects, but found that a higher anthropomorphism of the $\mathrm{VH}$ resulted in a reduced sense of social and co-presence, which conflicted with their hypothesis. They explained this result by stating that a more anthropomorphic image might reinforce the participant's expectations about realistic behaviors of the $\mathrm{VH}$ which could not be entirely met in the experiment. Chuah et al. [Chuah et al. 2013] approached this issue by developing hybrid VHs with a partial physical body (mannequin legs) in a medical application and concluded that increasing the physicality of VHs could encourage higher social presence.

Kim et al. [Kim et al. 2016] found while investigating plausible social interactions between participants and a VH that their extroverted participants reached a higher sense of social and copresence than their introverted participants. These results underline 
the effects that interpersonal differences can have on the results in social presence studies, which is one reason why we decided on a within-subject design in our experiment in this paper.

\section{EXPERIMENT}

In this section we describe our experiment to investigate a sense of copresence with a $\mathrm{VH}$ while interacting with the $\mathrm{VH}$ in a shared AR space. We varied the occurrence of visual conflicts caused by the VH's disregard for the rules of physicality (the dual occupancy of the $\mathrm{VH}$ with physical objects).

\subsection{Experimental Design}

We used a within-subjects design with two conditions (see Figure 2):

- No Conflict (“NC”): Participants experience that the VH avoids collisions with physical objects, for example, entering a room through an open door and moving only where no physical objects are present.

- Conflict (“CF”): Participants experience that the VH passes through physical objects, for example, entering a room by passing through a closed door and passing through physical tables while moving around in the room.

Between these conditions we only varied occurrences of the VH's spatial conflict with physical objects, i.e., the simultaneous occupancy of a space by both the $\mathrm{VH}$ and a physical object, but we maintained correct occlusions of the VH's body by physical objects, as would naturally be supported by the rendering. For example, one could not see the $\mathrm{VH}$ if a physical object (such as a wall or table) was in front of the VH. Participants experienced both of the conditions one after another in a counter-balanced order and their perceptions and behaviors were measured during and after each experience. Two (twin) VHs, "Sarah" and "Katie", which had an identical appearance except for the color of their shirt, were used to help participants to perceive the $\mathrm{VH}$ as a different character in each condition. We counter-balanced which $\mathrm{VH}$ was used in which experimental condition. Based on the analogy to our experience in the real world, we developed the following hypothesis about participant's perceived sense of copresence with the VH:

H-Co If a real human sees a virtual human follow the rules of physicality during interaction, it will lead to a higher self-reported sense of social presence and copresence than otherwise.

In order to provide a reasonably meaningful social interaction with the $\mathrm{VH}$ during the experiment, we prepared a brief verbal conversation, in which the $\mathrm{VH}$ asked four A/B type questions to assess the participant's personality for each condition, and the participants answered the questions by choosing either the option A or B that best described their personality [Myers 1962]. During the conversation, the $\mathrm{VH}$ moved around the room following the experimental conditions described above. In the middle of interaction, the $\mathrm{VH}$ asked the participant to hang a physical shirt on a physical coatrack across the room, while the VH blocked the path to the coatrack We observed how the participants behaved in this situation, i.e., whether they walked around the $\mathrm{VH}$, and thus tried to avoid a visual conflict, or walked straight through the VH.
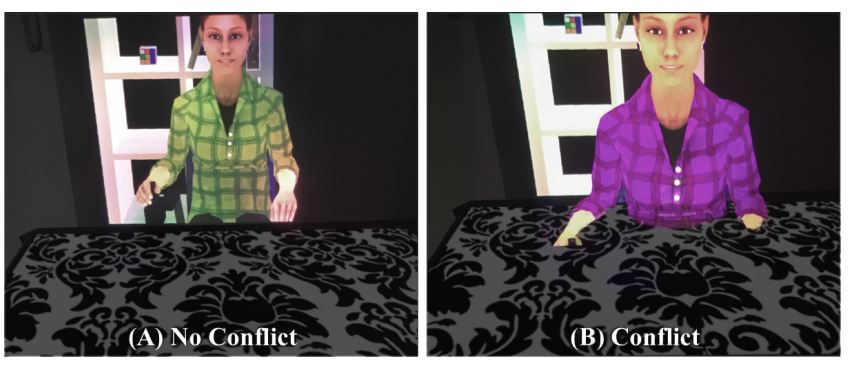

Figure 2: Study conditions: (A) the No Conflict ("NC") condition where the VH avoids physical collisions and (B) the Conflict ("CF") condition where the $\mathrm{VH}$ passes through physical objects.

\subsection{Material}

3.2.1 Physical Environment and Recordings. We furnished the experiment space with a chair, two tables, a shirt, a picture frame, and a coatrack (see Figure 3 ). The room with a size of $3.89 \mathrm{~m}$ by $3.89 \mathrm{~m}$ had two doors on its opposite sides, and the tables were placed across the middle of the room horizontally to the wall. The participants were instructed to sit on a chair where they could see the VH entering the room through one of the doors. The participant's behavior was captured by two webcams on the ceiling throughout the experiment, and we logged the HoloLens' position and orientation for examining the participant's movement trajectory in the laboratory room.

3.2.2 Virtual Humans and Human Controller. The two VHs in our experiment ("Sarah" and "Katie") could perform simple facial expressions, speech, and body gestures. To reduce the potential side effects of erratic body movements, we positioned the $\mathrm{VH}$ in a virtual electric wheelchair, i.e., she appeared to be physically challenged, and never stood up during the experiment (see Figure 1). The VH was displayed through a Microsoft HoloLens HMD, which was partially covered by a black polyether foam (see Figure 3 ). The reason for the foam was because of the HoloLens's narrow field of view-the VH's body could appear to be cropped at the edge of the small display when the participants were changing their view direction; this could possibly cause a severe distraction or break in presence for participants, which we thus avoided.

The VH was remotely controlled by a researcher who triggered the VH's pre-defined speech and behavioral animations. Therefore, we implemented a client-server application communicating between the HoloLens and the control workstation wirelessly. The application was implemented based on the Unity3D engine. The VH's voice had a spatial audio effect; hence, participants could feel the localized sound coming from the $\mathrm{VH}$ in the shared AR space. Throughout the interaction, the VH maintained a neutral or slightly pleased facial expression.

\subsection{Participants and Procedure}

We recruited 20 participants from our university community (ten males and ten females; age $M=24.1, S D=7.8$ ). The participants received a monetary compensation for their participation. 


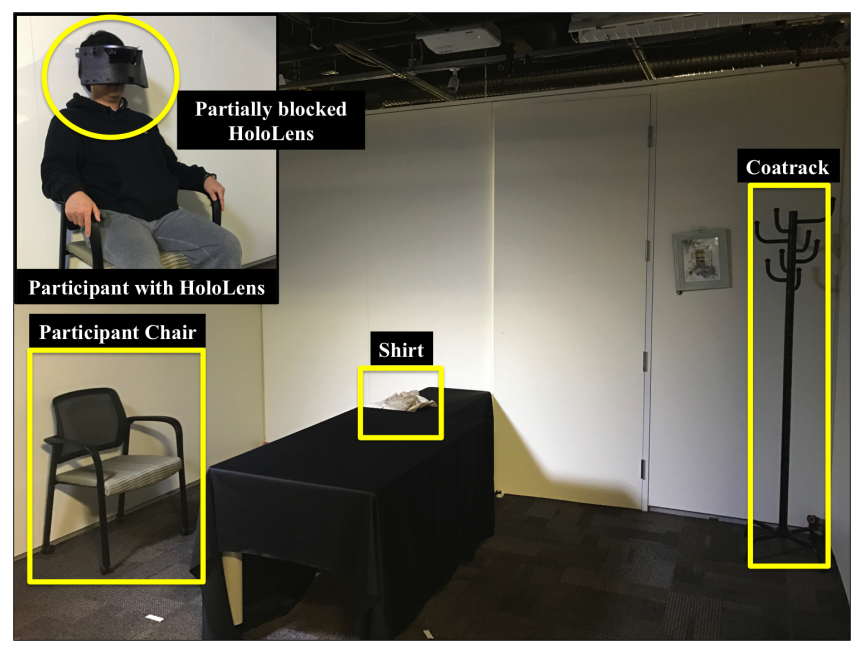

Figure 3: Experiment space and participant with the partially covered HoloLens HMD. Two tables in the middle of the room dividing half of the room, and the $\mathrm{VH}$ and the participant have a conversation across the tables. A coatrack is placed in the corner of the room next to a picture frame. A shirt is placed on one of the tables to investigate the participant's walking path around the $\mathrm{VH}$ towards the coatrack during the interaction.

When participants arrived, the experimenter asked them to read an informed consent form and to fill out a demographics questionnaire. The experimenter measured their interpupillary distance (IPD), and configured the HoloLens appropriately. Next, they were guided to the experimental room and instructed to sit in a chair They were informed that they were meeting twin VHs that had identical appearance one after another, and the VHs would ask a few A/B type questions related to the participant's personality. Once the participant wore the HoloLens, they had a couple of minutes to look around and make sure they saw virtual and physical objects in place-a virtual bookshelf, two real tables, a shirt, a coatrack, a photo frame on the wall (see Figure 3). The experimenter asked them to look toward the door so that the participant could observe the moment when the VH entered the room. When the experimenter left, depending on the experimental condition (see Section 3.1) the door was either closed or left opened, and the VH entered the room through the open door or passed through the closed door. While having a conversation to assess the participant's personality with the A/B type questions, the $\mathrm{VH}$ moved around the room, either avoiding physical collisions with the tables, or passing through the tables. Then, the $\mathrm{VH}$ asked the participant to put a shirt (on the table) on the coatrack in the corner of the room, while she placed herself in the path to the coatrack. Thus, participants had to decide whether they would avoid or pass through her. After the interaction, the participant was guided to leave the room through another door, which was not used by the $\mathrm{VH}$, and completed a post-questionnaire. Once participants had completed the post-questionnaire, they were guided to back into the room for the interaction with the other $\mathrm{VH}$ in the other experimental condition. All the participants experienced both of the experimental conditions during the experiment. Finally, participants were debriefed about their perception of and behavior with the $\mathrm{VH}$, and ended the study with receiving a monetary compensation. The total duration of the experiment per participant was approximately one hour including a brief discussion after the experiment.

\subsection{Dependent Variables}

Different subjective questionnaires have been introduced to measure social and copresence with VHs [Bailenson et al. 2003; Basdogan et al. 2000; Nowak 2001]. These questionnaires usually cover and combine multiple aspects together, such as a sense of copresence (i.e., being together in the same place), a degree of social connection (i.e., how closely they communicate/interact with each other), and a sense of realism (i.e., the VH's human-likeness). While such a combined questionnaire is beneficial when the goal is to measure the overall human perception of the $\mathrm{VH}$, it does not normally emphasize the aspect of the VH's interactivity with the surrounding physical environment and objects, which is important when trying to assess the sense of copresence with a $\mathrm{VH}$ in a shared AR space.

In Appendix A, we present a questionnaire combining our questions with relevant questions extracted and modified from three existing social and copresence questionnaires [Bailenson et al. 2003; Basdogan et al. 2000; Nowak 2001], to measure the perceived sense of a VH's ability to sense the real world, realism, physicality/interactivity in the physical space, and copresence.

3.4.1 Physicality and Interactivity in Physical Space. We prepared a set of eight questions measuring the perceived sense of the VH's physicality, the degree of perception that the VH exists in the physical space, and its interactivity with the environment. The interactivity of a $\mathrm{VH}$ with the physical environment is an important characteristic which is specific to AR environments.

3.4.2 Copresence. We prepared five questions-including two questions extracted from Bailenson et al. [Bailenson et al. 2003], one from Basdogan et al. [Basdogan et al. 2000], and two own questionto measure the sense of copresence with the $\mathrm{VH}$.

3.4.3 Sense. We thought participants could differently perceive the VH's ability to sense physical entities in the real world due to their observation of the VH's behavior passing through the surrounding physical objects. Thus, we prepared five questions evaluating the participant's perception of the VH's sensing ability via the five modalities hear, smell, see, touch, and taste.

3.4.4 Realism. We prepared seven questions on the participant's perception of the VH's realism, i.e., if it is perceived as a real human. We extracted several questions from existing questionnaires: one from Nowak [Nowak 2001], three from Bailenson et al. [Bailenson et al. 2003], and three from Basdogan et al. [Basdogan et al. 2000].

3.4.5 Godspeed Questionnaire. We also employed the "Godspeed" questionnaire from Bartneck et al. [Bartneck et al. 2009], which measures the four categories: anthropomorphism, animacy, likeability, and perceived intelligence. We expected that the responses for these categories would be generally more positive in the condition without a conflict. 
Table 1: Paired samples t-tests results and descriptives for the variables in our questionnaire (see Appendix A).

\begin{tabular}{|c|c|c|c|c|c|}
\hline Main Responses (t-tests) & $\mathbf{t}$ & df & $\mathbf{p}$ & \multicolumn{2}{|c|}{ Cohen's d } \\
\hline Sense & -1.978 & 19 & 0.063 & \multicolumn{2}{|c|}{-0.442} \\
\hline Realism & -1.509 & 19 & 0.148 & \multicolumn{2}{|c|}{-0.338} \\
\hline Physicality & -3.524 & 19 & $0.002^{* *}$ & \multicolumn{2}{|c|}{-0.788} \\
\hline Copresence & -2.253 & 19 & $0.036^{*}$ & \multicolumn{2}{|c|}{-0.504} \\
\hline \multicolumn{2}{|c|}{ Main Responses (descriptives) } & Group & $\mathbf{N}$ & Mean & SD \\
\hline \multirow{2}{*}{\multicolumn{2}{|c|}{ Sense }} & $\mathrm{NC}$ & 20 & 3.300 & 1.476 \\
\hline & & $\mathrm{CF}$ & 20 & 2.730 & 1.206 \\
\hline \multirow{2}{*}{\multicolumn{2}{|c|}{ Realism }} & $\mathrm{NC}$ & 20 & 3.457 & 1.457 \\
\hline & & $\mathrm{CF}$ & 20 & 3.064 & 1.083 \\
\hline \multirow{2}{*}{\multicolumn{2}{|c|}{ Physicality }} & $\mathrm{NC}$ & 20 & 3.956 & 1.218 \\
\hline & & $\mathrm{CF}$ & 20 & 3.319 & 1.363 \\
\hline \multirow{2}{*}{\multicolumn{2}{|c|}{ Copresence }} & NC & 20 & 4.708 & 1.223 \\
\hline & & $\mathrm{CF}$ & 20 & 4.133 & 1.169 \\
\hline
\end{tabular}

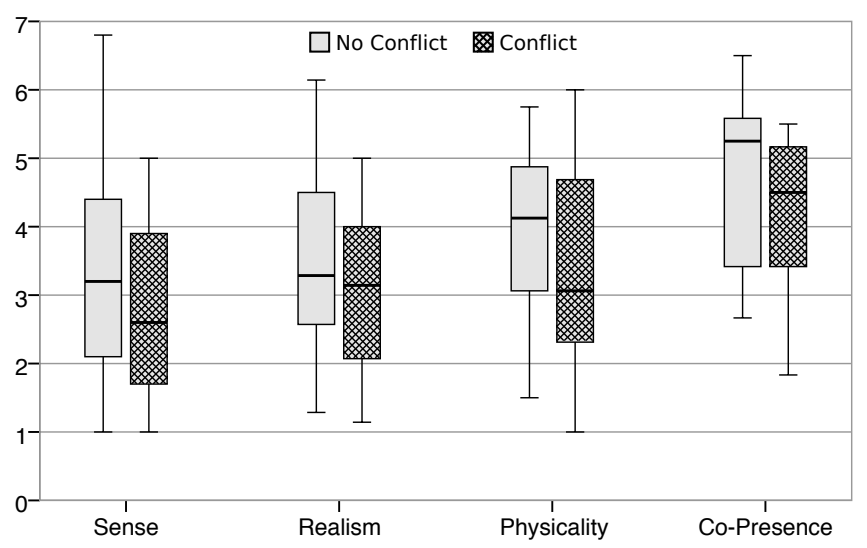

Figure 4: Box plots showing the results for the variables Sense, Realism, Physicality and Copresence.

\section{RESULTS}

In this section we present the subjective responses and participants' avoidance behavior in the experiment.

\subsection{Subjective Responses}

We decided to use parametric statistical tests to analyze the Likert scale data [Likert 1932] from the questionnaire, which has been shown to provide a valid method for the analysis of such ordinal data [Blaikie 2003; Knapp 1990]. We conducted paired samples ttests at the $\alpha=.05$ significance level to compare the responses within the participants for all subjective measures (averaged 7-point Likert-style scores) in the questionnaires.

Table 1 and Figure 4 show the descriptive and inferential statistical results for "Sense", "Realism", "Physicality" and "Copresence" as main responses. Table 2 and Figure 5 show the results for the Godspeed questionnaire.

4.1.1 Physicality and Interactivity in Physical Space. We found a significant difference between the conditions in the questions focusing on the VH's physicality and interactivity with the surrounding
Table 2: Paired samples t-tests results and descriptives for the Godspeed questions.

\begin{tabular}{|c|c|c|c|c|c|}
\hline Godspeed (t-tests) & $\mathbf{t}$ & df & $\mathbf{p}$ & \multicolumn{2}{|c|}{ Cohen's d } \\
\hline Anthropomorphism & 1.62 & 19 & 0.121 & \multicolumn{2}{|c|}{-0.363} \\
\hline Animacy & -2.491 & 19 & \multicolumn{2}{|c|}{$0.022^{*}$} & -0.557 \\
\hline Likeability & -1.651 & 19 & 0.115 & \multicolumn{2}{|c|}{-0.369} \\
\hline Perceived Intelligence & -1.967 & 19 & 0.064 & \multicolumn{2}{|c|}{-0.440} \\
\hline \multicolumn{2}{|c|}{ Godspeed (descriptives) } & Group & $\mathbf{N}$ & Mean & SD \\
\hline \multirow{2}{*}{\multicolumn{2}{|c|}{ Anthropomorphism }} & $\mathrm{NC}$ & 20 & 2.960 & 0.886 \\
\hline & & $\mathrm{CF}$ & 20 & 2.600 & 1.032 \\
\hline \multirow{2}{*}{\multicolumn{2}{|c|}{ Animacy }} & $\mathrm{NC}$ & 20 & 3.325 & 0.773 \\
\hline & & $\mathrm{CF}$ & 20 & 3.058 & 0.871 \\
\hline \multirow{2}{*}{\multicolumn{2}{|c|}{ Likeability }} & NC & 20 & 4.410 & 0.568 \\
\hline & & $\mathrm{CF}$ & 20 & 4.250 & 0.808 \\
\hline \multirow{2}{*}{\multicolumn{2}{|c|}{ Perceived Intelligence }} & $\mathrm{NC}$ & 20 & 4.030 & 0.694 \\
\hline & & $\mathrm{CF}$ & 20 & 3.730 & 0.857 \\
\hline
\end{tabular}

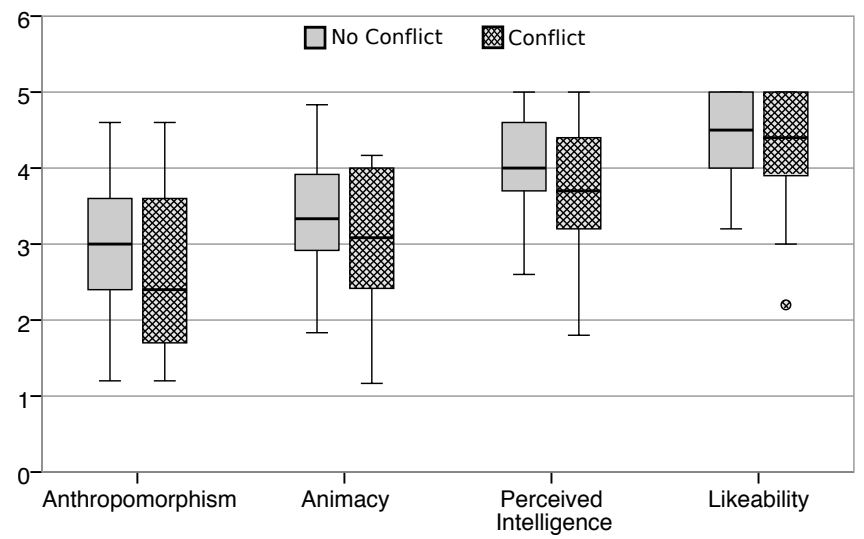

Figure 5: Box plots showing the results for the Godspeed questions in the two conditions.

physical space-the "NC" condition $(M=3.956, S D=1.218)$ and the "CF" condition $(M=3.319, S D=1.363) ; t(19)=-3.524, p=.002$. The results indicate that the visual conflicts caused by the VH's dual occupancy with physical objects had a negative impact on its perceived physicality.

4.1.2 Copresence. We found a significant difference in the participants' sense of copresence with the $\mathrm{VH}$ between the conditionsthe "NC" condition $(M=4.708, S D=1.223)$ and the "CF" condition $(M=4.133, S D=1.169) ; t(19)=-2.253, p=.036$. The estimated copresence was higher without visual conflicts.

4.1.3 Sense \& Realism. Although there was no significant difference in the "Sense" and the "Realism" variables, we observed a trend of higher scores in the "NC" condition compared to the "CF" condition for both variables.

4.1.4 Godspeed Questionnaire. For the "Godspeed" questionnaire, we found a significant difference in the participant's perceived animacy of the $\mathrm{VH}$ between the conditions-the "NC" condition $(M=3.325, S D=0.773)$ and the "CF" condition $(M=3.058$, 
$S D=0.871) ; t(19)=-2.491, p=.022$. Moreover, we observed a trend for a difference in the perceived intelligence of the $\mathrm{VH}$ between the conditions. Both of these showed higher scores in the condition without conflict.

\subsection{Avoidance Behavior}

We further examined the walking trajectory when participants were asked to move the shirt to the coatrack across the place where the VH was located (see Figure 3). Although participants were given this locomotion task twice due to the within-subjects design in our experiment, we only evaluated the first trial considering likely carryover effects between the first and second trial. Hence, we considered this as between-subject data based on the ten participants that started with the "NC" condition and the other ten participants that started with the "CF" condition. Figure 6 shows that more participants (4 out of 10) passed through the $\mathrm{VH}$ in the "NC" condition compared to those (1 out of 10$)$ in the "CF" condition. We looked at the recorded videos during the experiment and we confirmed that these participants would have collided with the body and/or wheelchair of the $\mathrm{VH}$ if it had been real.

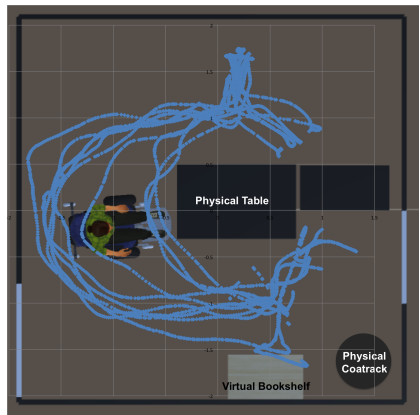

(A) No Conflict

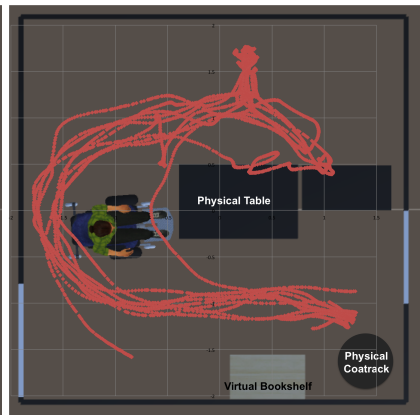

(B) Conflict
Figure 6: Trajectories of participants walking from one side of the room to the other: (A) blue lines indicate the "NC" condition and $(B)$ red lines indicate the "CF" condition.

\section{DISCUSSION}

The subjective responses provide clear support for our hypothesis $\mathrm{H}$-Co. When the participants did not see the $\mathrm{VH}$ passing through physical objects, they indicated a significantly higher sense of copresence, i.e., a sense of being together in the same place with the VH. They also attributed a significantly higher sense of physical existence to the VH. Moreover, the Godspeed questionnaire indicated that they attributed a significantly higher animacy, i.e. a sense of being alive and interactive, to the $\mathrm{VH}$ in the experiment. Also, the questionnaire results suggest a trend that participants might attribute a higher intelligence to the $\mathrm{VH}$, and a stronger belief that the VH could sense physical objects and events in the experiment.

The behavioral data, however, gives rise to different interpretations. Fewer participants passed through the VH after they had seen it pass through physical objects than otherwise. We would have expected that a higher sense of copresence would manifest itself via more natural locomotion behavior, i.e., avoiding collisions as is common among objects in the real world. However, our data might indicate a behavioral dynamics effect: Users who have seen a conflict might be more sensitized to avoid conflicts, whereas users who have never seen a conflict might not. We propose this as a hypothesis to be tested in future experiments.

In summary, such dual occupancy conflicts are an interesting challenge of real-virtual human perception and action in AR, and we predict that it will likely remain a persistent issue over the next years. It is a difficult question how a technological solution could avoid such conflicts without limiting real or virtual humans in their freedom to move and act in such a shared AR space.

\section{CONCLUSION}

In this paper we investigated the effects of the real-virtual spatial conflicts that can arise during social interaction between real and virtual humans in a shared AR space. The visual conflict which we call "dual occupancy," is caused by a virtual human occupying the same space as a physical object, or conversely, a real human occupying the same space as a virtual object. While it is generally assumed that such conflicts should be avoided, it is not always possible to do so from a technological point of view, without restricting the real or virtual human's freedom to move or act. We described a human-subject study in which we analyzed the effects of such conflicts on subjective estimates of copresence and perceived characteristics of the virtual human as well as the locomotion behavior of the participants. Our subjective responses support the premise that such conflicts reduce the sense of copresence and should be avoided if possible. However, our behavioral data suggests that avoiding such conflicts does not necessarily manifest itself in more natural locomotion behavior among the users. We even observed the opposite: Fewer participants in our experiment caused collisions with the virtual human after they had witnessed it causing collisions. We propose that future research should focus on evaluating such dynamics in real-virtual human interactions, which likely cannot be fully explained by having a high or low subjective sense of copresence, but might rather depend on more complex learned sensorimotor contingencies in AR interactions.

\section{ACKNOWLEDGMENTS}

The work presented in this publication is supported by the Office of Naval Research (ONR) Code 30 under Dr. Peter Squire, Program Officer (ONR awards N00014-14-1-0248 and N00014-12-1-1003). The authors would like to thank the members of the SREAL, UCF.

\section{REFERENCES}

Ronald T. Azuma. 1997. A Survey of Augmented Reality. Presence: Teleoperators and Virtual Environments 6, 4 (1997), 355-385.

Ronard T. Azuma and Gary Bishop. 1994. Improving Static and Dynamic Registration in an Optical See-Through HMD. In Computer Graphics (siggraph 94 conference proceedings ed.). ACM Press, Addison-Wesley, Orlando, FL USA, 197-204.

Jeremy N. Bailenson, Jim Blascovich, Andrew C. Beall, and Jack M. Loomis. 2003. Interpersonal distance in immersive virtual environments. Personality and Social Psychology Bulletin 29, 7 (2003), 819-833. https://doi.org/10.1177/0146167203029007002

Christoph Bartneck, Dana Kulić, Elizabeth Croft, and Susana Zoghbi. 2009. Measurement instruments for the anthropomorphism, animacy, likeability, perceived intelligence, and perceived safety of robots. International fournal of Social Robotics 1, 1 (2009), 71-81. https://doi.org/10.1007/s12369-008-0001-3

Cagatay Basdogan, Chih-Hao Ho, Mandayam A. Srinivasan, and Mel Slater. 2000. An experimental study on the role of touch in shared virtual environments. $A C M$ Transactions on Computer-Human Interaction 7, 4 (2000), 443-460. https://doi.org/ $10.1145 / 365058.365082$

Norman Blaikie. 2003. Analysing Quantitative Data. London: Sage Publications. 
Jim Blascovich. 2002. Social Influence within Immersive Virtual Environments. In The Social Life of Avatars, Ralph Schroeder (Ed.). Springer London, 127-145. https: //doi.org/10.1007/978-1-4471-0277-9_8

Jim Blascovich, Jack Loomis, Andrew C. Beall, Kimberly R. Swinth, Crystal L. Hoyt, and Jeremy N. Bailenson. 2002. Immersive virtual environment technology as a methodological tool for social psychology. Psychological Inquiry 13, 2 (apr 2002) 103-124. https://doi.org/10.1207/S15327965PLI1302_01

David E. Breen, Ross T. Whitaker, Eric Rose, and Mihran Tuceryan. 1996. Interactive occlusion and automatic object placement for augmented reality. In Computer Graphics Forum, Vol. 15. Wiley Online Library, 11-22.

Changhun Chae and Kwanghee Ko. 2008. Introduction of Physics Simulation in Augmented Reality. In 2008 International Symposium on Ubiquitous Virtual Reality. 37-40. https://doi.org/10.1109/ISUVR.2008.17

Joon Hao Chuah, Andrew Robb, Casey White, Adam Wendling, Samsun Lampotang, Regis Kopper, and Benjamin Lok. 2013. Exploring Agent Physicality and Social Presence for Medical Team Training. Presence: Teleoperators and Virtual Environments 22, 2 (2013), 141-170. https://doi.org/10.1162/PRES

Paul Debevec. 2005. Image-based Lighting. In Proceedings of the ACM Annual Conference on Computer Graphics and Interactive Techniques (SIGGRAPH) Courses. Article 3 , 26-34 pages.

Jesse Fox, Sun Joo Ahn, Joris H. Janssen, Leo Yeykelis, Kathryn Y. Segovia, and Jeremy N. Bailenson. 2014. Avatars Versus Agents: A Meta-Analysis Quantifying the Effect of Agency on Social Influence. Human-Computer Interaction 30, 5 (2014), 401-432. https://doi.org/10.1080/07370024.2014.921494

Chad Harms and Frank Biocca. 2004. Internal consistency and reliability of the networked minds measure of social presence. In Seventh Annual International Presence Workshop: Presence 2004. 246-251. http://cogprints.org/7026/

Thomas Holz, Abraham G. Campbell, Gregory M.P. O'Hare, John W. Stafford, Alan Martin, and Mauro Dragone. 2011. MiRA - Mixed Reality Agents. International fournal of Human-Computer Studies 69, 4 (apr 2011), 251-268. https://doi.org/10 1016/j.ijhcs.2010.10.001

Thomas Holz, Mauro Dragone, and Gregory M.P. O'Hare. 2009. Where robots and virtual agents meet: A survey of social interaction research across milgram's realityvirtuality continuum. International fournal of Social Robotics 1, 1 (2009), 83-93 https://doi.org/10.1007/s12369-008-0002-2

Dongsik Jo, Ki-Hong Kim, and Gerard Jounghyun Kim. 2015. SpaceTime: adaptive control of the teleported avatar for improved AR tele-conference experience. Computer Animation and Virtual Worlds 26 (2015), 259-269.

Kangsoo Kim, Gerd Bruder, Divine Maloney, and Greg Welch. 2016. The Influence of Real Human Personality on Social Presence with a Virtual Human in Augmented Reality. In Proceedings of the Combined International Conference on Artificial Reality \& Telexistence and Eurographics Symposium on Virtual Environments (ICAT-EGVE) $115-122$.

Thomas R. Knapp. 1990. Treating ordinal scales as interval scales: An attempt to resolve the controversy. Nurs. Res. 39 (1990), 121-123.

Rensis A. Likert. 1932. A technique for the measurement of attitudes. Arch. Psychol. 22 (1932), 5-55.

Jack M. Loomis and Joshua M. Knapp. 2003. Visual Perception of Egocentric Distance in Real and Virtual Environments. In Virtual and Adaptive Environments, L. Hettinger and M. Haas (Eds.). Erlbaum, 21-46.

Nadia Magnenat-Thalmann, George Papagiannakis, and Parag Chaudhuri. 2008. Applications of Interactive Virtual Humans in Mobile Augmented Reality. In Encyclopedia of Multimedia (2nd ed.), Borko Furht (Ed.). Springer, 362-368.

Microsoft. 2017a. Fragments. http://www.microsoft.com/microsoft-hololens/en-us/ apps/Fragments. (September 18 2017).

Microsoft. 2017b. Spatial Mapping (Windows Holographic). https://developer.microsoft com/en-us/windows/holographic/spatial_mapping. (September 18 2017)

Isabel Briggs Myers. 1962. The Myers-Briggs Type Indicator: Manual. Consulting Psychologists Press, Palo Alto. ii, 110 pages. http://trove.nla.gov.au/version/25681841

Kristine Nowak. 2001. Defining and Differentiating Copresence, Social Presence and Presence as Transportation. In International Workshop on Presence. http://citeseerx ist.psu.edu/viewdoc/summary?doi=10.1.1.19.5482

Kristine L. Nowak and Frank Biocca. 2003. The effect of the agency and anthropomorphism on users' sense of telepresence, copresence, and social presence in virtual environments. Presence: Teleoperators and Virtual Environments 12, 5 (2003), 481-494. http://www.mitpressjournals.org/doi/abs/10.1162/105474603322761289

Mohammad Obaid, Ionut Damian, Felix Kistler, Birgit Endrass, Johannes Wagner, and Elisabeth André. 2012. Cultural Behaviors of Virtual Agents in an Augmented Reality Environment. In International Conference on Intelligent Virtual Agents (Lecture Notes in Computer Science), Yukiko Nakano, Michael Neff, Ana Paiva, and Marilyn Walker (Eds.), Vol. 7502. Springer Berlin Heidelberg, 412-418. https://doi.org/10.1007/978-3-642-33197-8_42

Mohammad Obaid, Radosław Niewiadomski, and Catherine Pelachaud. 2011. Perception of Spatial Relations and of Coexistence with Virtual Agents. In International Conference on Intelligent Virtual Agents, HannesHögni Vilhjálmsson, Stefan Kopp, Stacy Marsella, and KristinnR. Thórisson (Eds.). Lecture Notes in Computer Science, Vol. 6895. Springer Berlin Heidelberg, 363-369. https://doi.org/10.1007/ 978-3-642-23974-8_39
Tomislav Pejsa, Julian Kantor, Hrvoje Benko, Eyal Ofek, and Andrew Wilson. 2016. Room2Room: Enabling Life-Size Telepresence in a Projected Augmented Reality Environment. In Proceedings of the 19th ACM Conference on Computer-Supported Cooperative Work \& Social Computing. ACM, 1716-1725.

Mel Slater. 2009. Place Illusion and Plausibility can Lead to Realistic Behaviour in Immersive Virtual Environments. Philosophical Transactions of the Royal Society of London. Series B, Biological Sciences 364, 1535 (dec 2009), 3549-3557. https: //doi.org/10.1098/rstb.2009.0138

Rémy Torre, Pascal Fua, Selim Balcisoy, Michal Ponder, and Daniel Thalmann. 2000. Interaction Between Real and Virtual Humans: Playing Checkers. In Virtual Environments (Eurographics Workshop). 23-32. https://doi.org/10.1007/978-3-7091-6785-4_

\section{APPENDIX}

\section{A QUESTIONNAIRES}

PH: Physicality and Interactivity in Physical Space

PH1. To what extent did you feel that the person and the virtual objects were still in the room after getting out of the room?

(1: They were no longer in the room., 7: They were in the room)

$\mathrm{PH}$ 2. I perceived that the person and the virtual objects were in a virtual world or a different dimension of space, which is not real. PH3. I felt the person was in the ___ space. (1: Virtual, 7: Real) PH4. I felt that the person was aware of the physical environment. PH5. I felt that the person could affect the physical environment.

PH6. I felt I could walk through the person.

PH7. I felt the person could walk through me.

PH8. The person seemed to have a physical body.

CP: Copresence (Sense of Being Together in the Same Place)

CP1. I perceived that I was in the presence of the person in the room with me.

CP2. I felt the person was watching me and was aware of my presence.

CP3. To what extent did you have a sense of being with the person?

CP4. To what extent was this like you were in the same room with the person?

CP5. I felt I was in the space. (1: Virtual, 7: Real)

${ }^{* *} \mathrm{CP} 5$ was scored by the difference with $\mathrm{PH} 3$.

\section{S: Perceived VH's Sensing Ability}

S1. I feel the person is able to hear if a fire alarm alerts.

S2. I feel the person is able to smell if I bake a bread.

S3. I feel the person is able to see if I show my family photo.

S4. I feel the person is able to touch if I give her my phone.

S5. I feel the person is able to taste if I bring a sandwich.

\section{R: VH Realism (Sense of Real Human)}

R1. To what extent does the person seem "real"? (1: Not real at all 7: Very real)

$\mathrm{R} 2$. The thought that the person is not a real person crosses my mind.

R3. The person appears to be sentient, conscious, and alive to me.

R4. I perceive the person as being only a computerized image, not as a real person.

R5. When you think back about your experience, do you remember this as more like just interacting with a computer or with a real person? (1: A computer, 7: A real person)

R6. To what extent was your experience with the person today like a previous real experience when you cooperatively worked together with another person? (e.g., lifting luggage, moving furniture, etc.)

(1: Not similar at all, 7: Very much similar)

R7. To what extent were there times, if at all, during which the computer interface seemed to vanish, and you were directly interacting with a real person? (1: I felt the computer interface all the time,

7: I was directly interacting with a real person) 